Kragujevac Journal of Mathematics

Volume 45(5) (2021), Pages 797-813.

\title{
HARDY-TYPE INEQUALITIES FOR AN EXTENSION OF THE RIEMANN-LIOUVILLE FRACTIONAL DERIVATIVE OPERATORS
}

\author{
SAJID IQBAL ${ }^{1}$, GHULAM FARID $^{2}$, JOSIP PEČARIĆ $^{3}$, AND ARTION KASHURI $^{4}$ \\ ABSTRACT. In this paper we present variety of Hardy-type inequalities and their \\ refinements for an extension of Riemann-Liouville fractional derivative operators. \\ Moreover, we use an extension of extended Riemann-Liouville fractional derivative \\ and modified extension of Riemann-Liouville fractional derivative using convex and \\ monotone convex functions. Furthermore, mean value theorems and $n$-exponential \\ convexity of the related functionals is discussed.
}

\section{INTRODUCTION}

The Hardy integral inequality is one of the most significant inequality in analysis with respect to its applications. In the recent years many researchers discover the new generalizations and refinements by involving fractional calculus operators (see $[1,4,16])$. Recently Iqbal et al. [8,9] study applications of Hardy-type and refined Hardy-type inequalities involving different kinds of fractional integral operators. Here we give such type of inequalities for more general forms of Riemann-Liouville fractional integral operators using convex and monotone convex functions.

Let $\left(\Sigma_{1}, \Omega_{1}, \mu_{1}\right)$ and $\left(\Sigma_{2}, \Omega_{2}, \mu_{2}\right)$ be measure spaces with positive $\sigma$-finite measures. Let $U(f, k)$ denote the class of functions $g: \Omega_{1} \rightarrow \mathbb{R}$ with the representation

$$
g(x)=\int_{\Omega_{2}} k(x, t) f(t) d \mu_{2}(t)
$$

and $A_{k}$ be an integral operator defined by

Key words and phrases. Inequalities, convex functions, Riemann-Liouville fractional derivative. 2010 Mathematics Subject Classification. Primary: 26D15. Secondary: 26D10, 26A33.

DOI 10.46793/KgJMat2105.797I

Received: August 17, 2018.

Accepted: May 21, 2019. 


$$
A_{k} f(x):=\frac{g(x)}{K(x)}=\frac{1}{K(x)} \int_{\Omega_{2}} k(x, t) f(t) d \mu_{2}(t),
$$

where $k: \Omega_{1} \times \Omega_{2} \rightarrow \mathbb{R}$ is measurable and non-negative kernel, $f: \Omega_{2} \rightarrow \mathbb{R}$ is measurable function and

$$
0<K(x):=\int_{\Omega_{2}} k(x, t) d \mu_{2}(t), \quad x \in \Omega_{1} .
$$

The following definition is presented in [13].

Definition 1.1. Let $I$ be an interval in $\mathbb{R}$. A function $\Phi: I \rightarrow \mathbb{R}$ is called convex if

$$
\Phi(\lambda x+(1-\lambda) y) \leq \lambda \Phi(x)+(1-\lambda) \Phi(y),
$$

for all points $x, y \in I$ and all $\lambda \in[0,1]$. The function $\Phi$ is strictly convex if inequality (1.3) holds strictly for all distinct points in $I$ and $\lambda \in(0,1)$.

The upcoming theorem is given in [11].

Theorem 1.1. Let $\left(\Omega_{1}, \Sigma_{1}, \mu_{1}\right)$ and $\left(\Omega_{2}, \Sigma_{2}, \mu_{2}\right)$ be measure spaces with positive $\sigma$ finite measures, $u$ be a weight function on $\Omega_{1}, k$ be a non-negative measurable function on $\Omega_{1} \times \Omega_{2}$ and $K$ be defined on $\Omega_{1}$ by (1.2). Suppose $K(x)>0$ for all $x \in \Omega_{1}$, that the function $x \mapsto u(x) \frac{k(x, t)}{K(x)}$ is integrable on $\Omega_{1}$ for each fixed $t \in \Omega_{2}$ and that $v$ is defined on $\Omega_{2}$ by

$$
v(t):=\int_{\Omega_{1}} u(x) \frac{k(x, t)}{K(x)} d \mu_{1}(x)<\infty .
$$

If $\Phi$ is a convex function on the interval $I \subseteq \mathbb{R}$, then the inequality

$$
\int_{\Omega_{1}} u(x) \Phi\left(A_{k} f(x)\right) d \mu_{1}(x) \leq \int_{\Omega_{2}} v(t) \Phi(f(t)) d \mu_{2}(t)
$$

holds for all measurable functions $f: \Omega_{2} \rightarrow \mathbb{R}$ such that $\operatorname{Im} f \subseteq I$, where $A_{k}$ is defined by (1.1).

Substitute $k(x, t)$ by $k(x, t) f_{2}(t)$ and $f$ by $\frac{f_{1}}{f_{2}}$, where $f_{i}: \Omega_{2} \rightarrow \mathbb{R}, i=1,2$, are measurable functions in Theorem 1.1, we obtain [6, Theorem 2.1].

Definition 1.2. Let $\Phi: I \rightarrow \mathbb{R}$ be a convex function, then the sub-differential of $\Phi$ in $x$ is denoted by $\partial \Phi(x)$ and is defined as

$$
\partial \Phi(x)=\{y \in \mathbb{R}: y \text { is the slope of a support line at } x\} .
$$

Next result is given in [4]. 
Theorem 1.2. Let the assumptions of Theorem 1.1 be satisfied. Moreover, if $\Phi$ is a convex function on an interval $I \subseteq \mathbb{R}$ and $\varphi: I \rightarrow \mathbb{R}$ is any function, such that $\varphi(x) \in \partial \Phi(x)$ for all $x \in \operatorname{Int} I$, then the inequality

$$
\begin{aligned}
& \int_{\Omega_{2}} v(t) \Phi(f(t)) d \mu_{2}(t)-\int_{\Omega_{1}} u(x) \Phi\left(A_{k} f(x)\right) d \mu_{1}(x) \\
\geq & \int_{\Omega_{1}} \frac{u(x)}{K(x)} \int_{\Omega_{2}} k(x, t)|| \Phi(f(t))-\Phi\left(A_{k} f(x)\right) \mid \\
& -\left|\varphi\left(A_{k} f(x)\right)\right| \cdot\left|f(t)-A_{k} f(x)\right| \mid d \mu_{2}(t) d \mu_{1}(x)
\end{aligned}
$$

holds for all measurable functions $f: \Omega_{2} \rightarrow \mathbb{R}$ such that $f(t) \in I$ for all $t \in \Omega_{2}$.

If $\Phi$ is a monotone convex function on an interval $I \subseteq \mathbb{R}$, then the inequality

$$
\begin{aligned}
& \int_{\Omega_{2}} v(t) \Phi(f(t)) d \mu_{2}(t)-\int_{\Omega_{1}} u(x) \Phi\left(A_{k} f(x)\right) d \mu_{1}(x) \\
\geq & \mid \int_{\Omega_{1}} \frac{u(x)}{K(x)} \int_{\Omega_{2}} \operatorname{sgn}\left(f(t)-A_{k} f(x)\right) k(x, t)\left[\Phi(f(t))-\Phi\left(A_{k} f(x)\right)\right. \\
& \left.-\left|\varphi\left(A_{k} f(x)\right)\right| \cdot\left(f(t)-A_{k} f(x)\right)\right] d \mu_{2}(t) d \mu_{1}(x) \mid,
\end{aligned}
$$

holds for all measurable functions $f: \Omega_{2} \rightarrow \mathbb{R}$ such that $f(t) \in I$ for all fixed $t \in \Omega_{2}$, where $A_{k} f$ is defined by (1.1).

Next mean value theorem is given in [5].

Theorem 1.3. Let $\left(\Omega_{1}, \Sigma_{1}, \mu_{1}\right),\left(\Omega_{2}, \Sigma_{2}, \mu_{2}\right)$ be measure spaces with $\sigma$-finite measures and $u: \Omega_{1} \rightarrow \mathbb{R}$ be a weight function. Let I be a compact interval of $\mathbb{R}, \tilde{h} \in C^{2}(I)$ and $f: \Omega_{2} \rightarrow \mathbb{R}$ a measurable function such that $\operatorname{Im} f \subseteq I$. Then there exists $\eta \in I$ such that

$$
\begin{aligned}
& \int_{\Omega_{2}} v(t) \tilde{h}(f(t)) d \mu_{2}(t)-\int_{\Omega_{1}} u(x) \tilde{h}\left(A_{k} f(x)\right) d \mu_{1}(x) \\
= & \frac{\tilde{h}^{\prime \prime}(\eta)}{2}\left[\int_{\Omega_{2}} v(t) f^{2}(t) d \mu_{2}(t)-\int_{\Omega_{1}} u(x)\left(A_{k} f(x)\right)^{2} d \mu_{1}(x)\right],
\end{aligned}
$$

where $A_{k} f$ and $v$ are defined by (1.1) and (1.4), respectively.

The definition of exponentially convex function is given in [3] by Bernstein.

Definition 1.3. A function $\Phi:(a, b) \rightarrow \mathbb{R}$ is exponentially convex if it is continuous and

$$
\sum_{i, j=1}^{n} t_{i} t_{j} \Phi\left(x_{i}+x_{j}\right) \geq 0,
$$

for all $n \in \mathbb{N}$ and all sequences $\left(t_{n}\right)_{n \in \mathbb{N}}$ and $\left(x_{n}\right)_{n \in \mathbb{N}}$ of real numbers, such that $x_{i}+x_{j} \in(a, b), 1 \leq i, j \leq n$. 
Lemma 1.1. Let $s \in \mathbb{R}$ and let the function $\varphi_{s}:(0, \infty) \rightarrow \mathbb{R}$ be defined by

$$
\varphi_{s}(x)= \begin{cases}\frac{x^{s}}{s(s-1)}, & s \neq 0,1, \\ -\log x, & s=0, \\ x \log x, & s=1 .\end{cases}
$$

Then $\varphi_{s}^{\prime \prime}(x)=x^{s-2}$, that is, $\varphi_{s}$ is a convex function.

The upcoming theorem is presented in [5].

Theorem 1.4. Let the conditions of Theorem 1.1 be satisfied and $\varphi_{s}$ be defined by (1.6). Let $f$ be a positive function. Then the function $\xi: \mathbb{R} \rightarrow[0, \infty)$ defined by

$$
\xi(s)=\int_{\Omega_{2}} v(t) \varphi_{s}(f(t)) d \mu_{2}(t)-\int_{\Omega_{1}} u(x) \varphi_{s}\left(A_{k} f(x)\right) d \mu_{1}(x)
$$

is exponentially convex.

Theorem 1.5. Let the conditions of Theorem 1.3 be satisfied. Moreover, $k, \tilde{h} \in C^{2}(I)$ such that $\tilde{h}^{\prime \prime}(x) \neq 0$ for every $x \in I$ and

$$
\int_{\Omega_{2}} v(t) \tilde{h}(f(t)) d \mu_{2}(t)-\int_{\Omega_{1}} u(x) \tilde{h}\left(A_{k} f(x)\right) d \mu_{1}(x) \neq 0 .
$$

Then there exists $\eta \in I$ such that it holds

$$
\frac{k^{\prime \prime}(\eta)}{\tilde{h}^{\prime \prime}(\eta)}=\frac{\int_{\Omega_{2}} v(t) k(f(t)) d \mu_{2}(t)-\int_{\Omega_{1}} u(x) k\left(A_{k} f(x)\right) d \mu_{1}(x)}{\int_{\Omega_{2}} v(t) \tilde{h}(f(t)) d \mu_{2}(t)-\int_{\Omega_{1}} u(x) \tilde{h}\left(A_{k} f(x)\right) d \mu_{1}(x)} .
$$

By Theorem 1.1, and bearing in mind (1.5), we define the following positive linear functional:

$$
\Delta(\Phi)=\int_{\Omega_{2}} v(t) \Phi(f(t)) d \mu_{2}(t)-\int_{\Omega_{1}} u(x) \Phi\left(A_{k} f(x)\right) d \mu_{1}(x) .
$$

Let $I \subseteq \mathbb{R}$ be an interval and $f: I \rightarrow \mathbb{R}$ be a function. Then for distinct points $z_{i} \in I, i=0,1,2$, the divided differences of first and second order are defined by

$$
\begin{aligned}
{\left[z_{i}, z_{i+1} ; f\right] } & =\frac{f\left(z_{i+1}\right)-f\left(z_{i}\right)}{z_{i+1}-z_{i}}, \quad i=0,1, \\
{\left[z_{0}, z_{1}, z_{2} ; f\right] } & =\frac{\left[z_{1}, z_{2} ; f\right]-\left[z_{0}, z_{1} ; f\right]}{z_{2}-z_{0}} .
\end{aligned}
$$

The values of the divided differences are independent of the order of points $z_{0}, z_{1}$, $z_{2}$ and may be extended to include the cases when some or all points are equal, that is $\left[z_{0}, z_{0} ; f\right]=\lim _{z_{1} \rightarrow z_{0}}\left[z_{0}, z_{1} ; f\right]=f^{\prime}\left(z_{0}\right)$, provided that $f^{\prime}$ exists.

Now, passing through the limit $z_{1} \rightarrow z_{0}$ and replacing $z_{2}$ by $z$ in (1.8), we have

$$
\left[z_{0}, z_{0}, z ; f\right]=\lim _{z_{1} \rightarrow z_{0}}\left[z_{0}, z_{1}, z ; f\right]=\frac{f(z)-f\left(z_{0}\right)-\left(z-z_{0}\right) f^{\prime}\left(z_{0}\right)}{\left(z-z_{0}\right)^{2}}, \quad z \neq z_{0}
$$


provided that $f^{\prime}$ exists. Also, passing to the limit $z_{i} \rightarrow z, i=0,1,2$, in (1.8), we have

$$
[z, z, z ; f]=\lim _{z_{i} \rightarrow z}\left[z_{0}, z_{1}, z_{2} ; f\right]=\frac{f^{\prime \prime}(z)}{2}
$$

provided that $f^{\prime \prime}$ exists.

One can observe that if for all $z_{0}, z_{1} \in I,\left[z_{0}, z_{1}, f\right] \geq 0$, then $f$ is increasing on $I$ and if for all $z_{0}, z_{1}, z_{2} \in I,\left[z_{0}, z_{1}, z_{2} ; f\right] \geq 0$, then $f$ is convex on $I$.

Next, we recall the notion of $n$-exponential convexity given in [15].

Definition 1.4. For any open interval $I$ of $\mathbb{R}$, the function $\Phi: I \rightarrow \mathbb{R}$ is $n$ exponentially convex in the Jensen sense on $I$ if

$$
\sum_{i, j=1}^{n} t_{i} t_{j} \Phi\left(\frac{\zeta_{i}+\zeta_{j}}{2}\right) \geq 0
$$

holds for all choices of $t_{i} \in \mathbb{R}, \zeta_{i} \in I, i=1, \ldots, n$.

A function $\Phi: I \rightarrow \mathbb{R}$ is $n$-exponentially convex on $I$ if it is $n$-exponentially convex in the Jensen sense and continuous on $I$.

The following theorem is given in [7].

Theorem 1.6. Let $\Gamma=\left\{\Phi_{p}: p \in J\right\}$ be a family of functions defined on $I$, such that the function $p \mapsto\left[z_{0}, z_{1}, z_{2} ; \Phi_{p}\right]$ is n-exponentially convex in the Jensen sense on $J$ for every three distinct points $z_{0}, z_{1}, z_{2} \in I$. Let $\Delta$ be linear functionals defined by (1.7). Then the function $p \mapsto \Delta\left(\Phi_{p}\right)$ is n-exponentially convex in the Jensen sense on $J$, if it is continuous on $J$.

\section{Hardy-Type Inequalities for Fractional Derivative}

We begin with the well known definition of Riemann-Liouville fractional derivative od order $\mu$ is defined $([10,19])$ by

$$
\mathfrak{D}_{x}^{\mu}\{f(x)\}=\frac{1}{\Gamma(-\mu)} \int_{0}^{x} f(t)(x-t)^{-\mu-1} d t, \quad \operatorname{Re}(\mu)>0 .
$$

For the case $m-1<\operatorname{Re}(\mu)<m, \operatorname{Re}(\mu)>0$, where $m=1,2, \ldots$, it follows

$$
\mathfrak{D}_{x}^{\mu}\{f(x)\}=\frac{d^{m}}{d x^{m}} \mathfrak{D}_{x}^{\mu-m}\{f(x)\}=\frac{d^{m}}{d x^{m}}\left\{\frac{1}{\Gamma(-\mu+m)} \int_{0}^{x} f(t)(x-t)^{-\mu+m-1} d t\right\}
$$

and

$$
\mathfrak{D}_{x}^{\mu}\left\{x^{\sigma}\right\}=\frac{\Gamma(\sigma+1)}{\Gamma(\sigma-\mu+1)} x^{\sigma-\mu}, \quad \operatorname{Re}(\sigma)>-1 .
$$

The extended Riemann-Liouville fractional derivative of order $\mu$ is defined in [14] by

$$
\mathfrak{D}_{x}^{\mu}\{f(x) ; p\}=\frac{1}{\Gamma(-\mu)} \int_{0}^{x} f(t)(x-t)^{-\mu-1} \exp \left(-\frac{p x^{2}}{t(x-t)}\right) d t, \quad \operatorname{Re}(\mu)>0
$$


For the case $m-1<\operatorname{Re}(\mu)<m$, where $m=1,2, \ldots$, it follows

$$
\begin{aligned}
& \mathfrak{D}_{x}^{\mu}\{f(x) ; p\}=\frac{d^{m}}{d x^{m}} \mathfrak{D}_{x}^{\mu-m}\{f(x) ; p\} \\
= & \frac{d^{m}}{d x^{m}}\left\{\frac{1}{\Gamma(-\mu+m)} \int_{0}^{x} f(t)(x-t)^{-\mu+m-1} \exp \left(-\frac{p x^{2}}{t(x-t)}\right) d t\right\}, \quad \operatorname{Re}(\mu)>0 .
\end{aligned}
$$

An extension of fractional derivative operator established in [2] is given by

$$
\mathfrak{D}_{x}^{\mu}\{f(x) ; p, q\}=\frac{1}{\Gamma(-\mu)} \int_{0}^{x} f(t)(x-t)^{-\mu-1} \exp \left(-\frac{p x}{t}-\frac{q x}{(x-t)}\right) d t, \quad \operatorname{Re}(\mu)>0 .
$$

For example

$$
\mathfrak{D}_{x}^{\mu}\{f(x) ; p, q\}_{x=1}=\frac{B_{p, q}(\nu+1, \mu)}{\Gamma(-\mu)},
$$

where $B_{p, q}(\nu+1, \mu)$ is the extended beta functions (see [12]) defined by

$$
B_{p, q}(x, y)=\int_{0}^{1} t^{x-1}(1-t)^{y-1} e^{-\frac{p}{t}-\frac{q}{1-t}} d t, \quad x, y, p, q \in \mathbb{C}, \operatorname{Re}(p)>0, \operatorname{Re}(q)>0 .
$$

For $p=q$ we denote $B_{p, q}$ by $B_{p}$ and for $p=q=0$ we get the classical beta function defined by

$$
B(x, y)=\int_{0}^{1} t^{x-1}(1-t)^{y-1} d t, \quad \operatorname{Re}(x)>0, \operatorname{Re}(y)>0 .
$$

Theorem 2.1. Let $\operatorname{Re}(p)>0, \operatorname{Re}(q)>0$ and $\operatorname{Re}(\mu)>0$. Let $\mathfrak{D}_{x}^{\mu}\{f(x) ; p, q\}$ denotes the extension of Riemann-Liouville fractional derivative of order $\mu$ and let $u$ be $a$ weight function defined on $(0, b)$. For each fixed $t \in(0, b)$, define a function $\tilde{v}$ by

$$
\tilde{v}(t)=\int_{t}^{b} u(x) \frac{(x-t)^{-\mu-1} \exp \left(-\frac{p x}{t}-\frac{q x}{(x-t)}\right)}{x^{-\mu} B_{p, q}(1,-\mu)} d x<\infty .
$$

If $\Phi$ is a convex function on the interval $I \in \mathbb{R}$, then the inequality

$$
\int_{0}^{b} u(x) \Phi\left(\frac{\Gamma(-\mu) x^{\mu} \mathfrak{D}_{x}^{\mu}\{f(x) ; p, q\}}{B_{p, q}(1,-\mu)}\right) d x \leq \int_{0}^{b} \tilde{v}(t) \Phi(f(t)) d t
$$

holds true for all measurable functions $f \in L(a, b)$.

Proof. Applying Theorem 1.1 with $\Omega_{1}=\Omega_{2}=(0, b), d \mu_{1}(x)=d x, d \mu_{2}(t)=d t$,

$$
\tilde{k}(x, t)= \begin{cases}\frac{1}{\Gamma(-\mu)}(x-t)^{-\mu-1} \exp \left(-\frac{p x}{t}-\frac{q x}{(x-t)}\right), & 0 \leq t \leq x, \\ 0, & x<t \leq b\end{cases}
$$




$$
\tilde{K}(x)=\frac{1}{\Gamma(-\mu)} \int_{0}^{x}(x-t)^{-\mu-1} \exp \left(-\frac{p x}{t}-\frac{q x}{(x-t)}\right) d t=\frac{x^{-\mu} B_{p, q}(1,-\mu)}{\Gamma(-\mu)}
$$

and

$$
A_{k} f(x)=\frac{\Gamma(-\mu) x^{\mu} \mathfrak{D}_{x}^{\mu}\{f(x) ; p, q\}}{B_{p, q}(1,-\mu)}
$$

we get inequality (2.7).

Substitute $\tilde{k}(x, t)$ by $\tilde{k}(x, t) f_{2}(t)$ and $f$ by $\frac{f_{1}}{f_{2}}$, where $f_{i}: \Omega_{2} \rightarrow \mathbb{R}, i=1,2$, are measurable functions in Theorem 2.1 we obtain the following result.

Theorem 2.2. Let $\operatorname{Re}(p)>0, \operatorname{Re}(q)>0$ and $\operatorname{Re}(\mu)>0$. Let $\mathfrak{D}_{x}^{\mu}\{f(x) ; p, q\}$ denotes the extension of Riemann-Liouville fractional derivative of order $\mu$ and let $u$ be a weight function defined on $(0, b)$. For each fixed $t \in(0, b)$, define a function

$$
\tilde{p}(t):=\frac{f_{2}(t)}{\Gamma(-\mu)} \int_{t}^{b} u(x) \frac{(x-t)^{-\mu-1} \exp \left(-\frac{p x}{t}-\frac{q x}{(x-t)}\right)}{\mathfrak{D}_{x}^{\mu}\left\{f_{2}(x) ; p, q\right\}} d x<\infty .
$$

If $\Phi: I \rightarrow \mathbb{R}$ is a convex function and $\frac{\mathfrak{D}_{x}^{\mu}\left\{f_{1}(x) ; p, q\right\}}{\mathfrak{D}_{x}^{\mu}\left\{f_{2}(x) ; p, q\right\}}, \frac{f_{1}(t)}{f_{2}(t)} \in I$, then the inequality

$$
\int_{0}^{b} u(x) \Phi\left(\frac{\mathfrak{D}_{x}^{\mu}\left\{f_{1}(x) ; p, q\right\}}{\mathfrak{D}_{x}^{\mu}\left\{f_{2}(x) ; p, q\right\}}\right) d x \leq \int_{0}^{b} \tilde{p}(t) \Phi\left(\frac{f_{1}(t)}{f_{2}(t)}\right) d t
$$

holds true.

New refined weighted Hardy-type inequality for extension of Riemann-Liouville fractional derivative (2.5) is given in the next theorem.

Theorem 2.3. Let the assumptions of Theorem 2.1 be satisfied. Moreover, if $\Phi$ is a convex function on an interval $I \subseteq \mathbb{R}$ and $\varphi: I \rightarrow \mathbb{R}$ is any function, such that $\varphi(x) \in \partial \Phi(x)$ for all $x \in \operatorname{Int} I$, then the inequality

$$
\begin{aligned}
& \int_{0}^{b} \tilde{v}(t) \Phi(f(t)) d t-\int_{0}^{b} u(x) \Phi\left(\frac{\Gamma(-\mu) x^{\mu} \mathfrak{D}_{x}^{\mu}\{f(x) ; p, q\}}{B_{p, q}(1,-\mu)}\right) d x \\
\geq & \int_{0}^{b} \frac{u(x)}{x^{-\mu} B_{p, q}(1,-\mu)} \int_{0}^{x}(x-t)^{-\mu-1} \exp \left(-\frac{p x}{t}-\frac{q x}{(x-t)}\right) \\
& \times \| \Phi(f(t))-\Phi\left(\frac{\Gamma(-\mu) x^{\mu} \mathfrak{D}_{x}^{\mu}\{f(x) ; p, q\}}{B_{p, q}(1,-\mu)}\right) \mid \\
& -\left|\varphi\left(\frac{\Gamma(-\mu) x^{\mu} \mathfrak{D}_{x}^{\mu}\{f(x) ; p, q\}}{B_{p, q}(1,-\mu)}\right)\right|\left|f(t)-\frac{\Gamma(-\mu) x^{\mu} \mathfrak{D}_{x}^{\mu}\{f(x) ; p, q\}}{B_{p, q}(1,-\mu)}\right| \mid d t d x
\end{aligned}
$$


holds for all measurable functions $f: \Omega_{2} \rightarrow \mathbb{R}$. If $\Phi$ is a monotone convex function on an interval $I \subseteq \mathbb{R}$, then the inequality

$$
\begin{aligned}
& \int_{0}^{b} \tilde{v}(t) \Phi(f(t)) d t-\int_{0}^{b} u(x) \Phi\left(\frac{\Gamma(-\mu) x^{\mu} \mathfrak{D}_{x}^{\mu}\{f(x) ; p, q\}}{B_{p, q}(1,-\mu)}\right) d x \\
\geq & \mid \int_{0}^{b} \frac{u(x)}{x^{-\mu} B_{p, q}(1,-\mu)} \int_{0}^{x} \operatorname{sgn}\left(f(t)-\frac{\Gamma(-\mu) x^{\mu} \mathfrak{D}_{x}^{\mu}\{f(x) ; p, q\}}{B_{p, q}(1,-\mu)}\right) \\
& \times(x-t)^{-\mu-1} \exp \left(-\frac{p x}{t}-\frac{q x}{(x-t)}\right)\left[\Phi(f(t))-\Phi\left(\frac{\Gamma(-\mu) x^{\mu} \mathfrak{D}_{x}^{\mu}\{f(x) ; p, q\}}{B_{p, q}(1,-\mu)}\right)\right. \\
& \left.-\left|\varphi\left(\frac{\Gamma(-\mu) x^{\mu} \mathfrak{D}_{x}^{\mu}\{f(x) ; p, q\}}{B_{p, q}(1,-\mu)}\right)\right|\left(f(t)-\frac{\Gamma(-\mu) x^{\mu} \mathfrak{D}_{x}^{\mu}\{f(x) ; p, q\}}{B_{p, q}(1,-\mu)}\right)\right] d t d x \mid
\end{aligned}
$$

holds for all measurable functions $f:(0, b) \rightarrow \mathbb{R}$.

Proof. Similar to Theorem 2.1 by applying Theorem 1.2 .

Next we give the mean value theorems for extension of Riemann-Liouville fractional derivative of order $\mu$.

Theorem 2.4. Let the assumptions of Theorem 2.1 be satisfied. Let I be a compact interval of $\mathbb{R}, \tilde{h} \in C^{2}(I)$ and $f:(0, b) \rightarrow \mathbb{R}$ a measurable function such that $\operatorname{Im} f \subseteq I$. Then there exists $\eta \in I$ such that

$$
\begin{aligned}
& \int_{0}^{b} \tilde{v}(t) \tilde{h}(f(t)) d t-\int_{0}^{b} u(x) \tilde{h}\left(\frac{\Gamma(-\mu) x^{\mu} \mathfrak{D}_{x}^{\mu}\{f(x) ; p, q\}}{B_{p, q}(1,-\mu)}\right) d x \\
= & \frac{\tilde{h}^{\prime \prime}(\eta)}{2}\left[\int_{0}^{b} \tilde{v}(t) f^{2}(t) d t-\int_{0}^{b} u(x)\left(\frac{\Gamma(-\mu) x^{\mu} \mathfrak{D}_{x}^{\mu}\{f(x) ; p, q\}}{B_{p, q}(1,-\mu)}\right)^{2} d x\right],
\end{aligned}
$$

where $\tilde{v}$ is defined by (2.6).

Proof. Similar to proof of Theorem 2.1, by applying Theorem 1.3.

Theorem 2.5. Let the assumptions of Theorem 2.4 be satisfied. Moreover, $k, \tilde{h} \in$ $C^{2}(I)$ such that $\tilde{h}^{\prime \prime}(x) \neq 0$ for every $x \in I$ and

$$
\int_{0}^{b} \tilde{v}(t) \tilde{h}(f(t)) d t-\int_{0}^{b} u(x) \tilde{h}\left(\frac{\Gamma(-\mu) x^{\mu} \mathfrak{D}_{x}^{\mu}\{f(x) ; p, q\}}{B_{p, q}(1,-\mu)}\right) d x \neq 0 .
$$

Then there exists $\eta \in I$ such that it holds

$$
\frac{k^{\prime \prime}(\eta)}{\tilde{h}^{\prime \prime}(\eta)}=\frac{\int_{0}^{b} \tilde{v}(t) k(f(t)) d t-\int_{0}^{b} u(x) k\left(\frac{\Gamma(-\mu) x^{\mu} \mathfrak{D}_{x}^{\mu}\{f(x) ; p, q\}}{B_{p, q}(1,-\mu)}\right) d x}{\int_{0}^{b} \tilde{v}(t) \tilde{h}(f(t)) d t-\int_{0}^{b} u(x) \tilde{h}\left(\frac{\Gamma(-\mu) x^{\mu} \mathfrak{D}_{x}^{\mu}\{f(x) ; p, q\}}{B_{p, q}(1,-\mu)}\right) d x} .
$$


Proof. Similar to proof of Theorem 2.1, by applying Theorem 1.5.

Theorem 2.6. Let the conditions of Theorem 2.1 be satisfied and $\varphi_{s}$ be defined by (1.6). Let $f$ be a positive function. Then the function $\xi: \mathbb{R} \rightarrow[0, \infty)$ defined by

$$
\xi(s)=\int_{0}^{b} \tilde{v}(t) \varphi_{s}(f(t)) d t-\int_{0}^{b} u(x) \varphi_{s}\left(\frac{\Gamma(-\mu) x^{\mu} \mathfrak{D}_{x}^{\mu}\{f(x) ; p, q\}}{B_{p, q}(1,-\mu)}\right) d x
$$

is exponentially convex.

Proof. Applying Theorem 1.4, with $\Omega_{1}=\Omega_{2}=(a, b), d \mu_{1}(x)=d x, d \mu_{2}(t)=d t$ and $\tilde{k}(x, t)$ given in $(2.8)$, we get the exponential convexity of linear functional $(2.10)$.

\section{Hardy-Type Inequalities for Extension of Extended Riemman-Liouvill Fractional Derivative}

Recently Rehaman et al. [17] define an extension of extended Riemman-Liouvill fractional derivative of order $\mu$ as

$$
\begin{aligned}
\mathfrak{D}_{x}^{\mu}\{f(x) ; p, q ; \lambda ; \rho\}= & \frac{1}{\Gamma(-\mu)} \int_{0}^{x} f(t)(x-t)^{-\mu-1}{ }_{1} F_{1}\left[\lambda ; \rho ;-\frac{p x}{t}\right] \\
& \times{ }_{1} F_{1}\left[\lambda ; \rho ;-\frac{q x}{(x-t)}\right] d t, \quad \operatorname{Re}(\mu)>0 .
\end{aligned}
$$

For the case $m-1<\operatorname{Re}(\mu)<m$, where $m=1,2, \ldots$, it follows

$$
\begin{aligned}
\mathfrak{D}_{x}^{\mu}\{f(x) ; p ; \lambda ; \rho\}= & \frac{d^{m}}{d x^{m}} \mathfrak{D}_{x}^{\mu-m}\{f(x) ; p ; q ; \lambda ; \rho\} \\
= & \frac{d^{m}}{d x^{m}}\left\{\frac{1}{\Gamma(-\mu+m)} \int_{0}^{x} f(t)(x-t)^{-\mu+m-1}\right. \\
& \left.\times{ }_{1} F_{1}\left[\lambda ; \rho ;-\frac{p x}{t}\right]{ }_{1} F_{1}\left[\lambda ; \rho ;-\frac{q x}{(x-t)}\right] d t\right\},
\end{aligned}
$$

where $\operatorname{Re}(\mu)>0, \operatorname{Re}(p)>0, \operatorname{Re}(q)>0$. It is clear that $\lambda=\rho$, then (3.1) reduces to $(2.5)$.

Theorem 3.1. Let $\operatorname{Re}(p)>0, \operatorname{Re}(q)>0, \operatorname{Re} \mu)>0, \operatorname{Re}(\lambda)>0$ and $\operatorname{Re}(\rho)>0$. Let $\mathfrak{D}_{x}^{\mu}\{f(x) ; p, q, \lambda, \rho\}$ be the extension of extended Riemman-Liouvill fractional derivative of order $\mu$. Let $u$ be a weight function defined on $(0, b)$, then $\bar{v}$ is defined by

$$
\bar{v}(t)=\int_{t}^{b} u(x) \frac{(x-t)^{-\mu-1}{ }_{1} F_{1}\left[\lambda ; \rho ;-\frac{p x}{t}\right]{ }_{1} F_{1}\left[\lambda ; \rho ;-\frac{q x}{(x-t)}\right]}{\int_{0}^{x}(x-t)^{-\mu-1}{ }_{1} F_{1}\left[\lambda ; \rho ;-\frac{p x}{t}\right]{ }_{1} F_{1}\left[\lambda ; \rho ;-\frac{q x}{(x-t)}\right]} d x<\infty .
$$


If $\Phi$ is a convex function on the interval $I$, then the inequality

$$
\int_{0}^{b} u(x) \Phi\left(\frac{\mathfrak{D}_{x}^{\mu}\{f(x) ; p, q ; \lambda ; \rho\}}{\bar{K}(x)}\right) d x \leq \int_{0}^{b} \bar{v}(t) \Phi(f(t)) d t
$$

holds true.

Proof. Applying Theorem 1.1, with $\Omega_{1}=\Omega_{2}=(0, b), d \mu_{1}(x)=d x, d \mu_{2}(t)=d t$,

$$
\begin{aligned}
& \bar{k}(x, t)= \begin{cases}\frac{1}{\Gamma(-\mu)}(x-t)^{-\mu-1}{ }_{1} F_{1}\left[\lambda ; \rho ;-\frac{p x}{t}\right]{ }_{1} F_{1}\left[\lambda ; \rho ;-\frac{q x}{(x-t)}\right], & 0 \leq t \leq x, \\
0, & x<t \leq b,\end{cases} \\
& \bar{K}(x)=\frac{1}{\Gamma(-\mu)} \int_{0}^{x}(x-t)^{-\mu-1}{ }_{1} F_{1}\left[\lambda ; \rho ;-\frac{p x}{t}\right]{ }_{1} F_{1}\left[\lambda ; \rho ;-\frac{q x}{(x-t)}\right],
\end{aligned}
$$

and $\bar{v}$ as in (3.2), we get inequality (3.3).

Substitute $\bar{k}(x, t)$ by $\bar{k}(x, t) f_{2}(t)$ and $f$ by $\frac{f_{1}}{f_{2}}$ where $f_{i}: \Omega_{2} \rightarrow \mathbb{R}, i=1,2$, are measurable functions in Theorem 3.1 we obtain the following result.

Theorem 3.2. Let $D_{x}^{\mu}\{f(x) ; p, q, \lambda, \rho\}$ be the fractional derivative operator of order $\mu$. Let $u$ be a weight function defined on $(0, b)$ and for each fixed $t \in(0, b)$ define $\bar{p}$ on $(0, b)$ as

$$
\bar{p}(t):=\frac{f_{2}(t)}{\Gamma(-\mu)} \int_{t}^{b} u(x) \frac{(x-t)^{-\mu-1}{ }_{1} F_{1}\left[\lambda ; \rho ;-\frac{p x}{t}\right]{ }_{1} F_{1}\left[\lambda ; \rho ;-\frac{q x}{(x-t)}\right]}{\mathfrak{D}_{x}^{\mu}\left\{f_{2}(x) ; p, q ; \lambda ; \rho\right\}(x)} d x<\infty .
$$

If $\Phi: I \rightarrow \mathbb{R}$ is a convex function, then the inequality

$$
\int_{0}^{b} u(x) \Phi\left(\frac{\mathfrak{D}_{x}^{\mu}\left\{f_{1}(x) ; p, q ; \lambda ; \rho\right\}}{\mathfrak{D}_{x}^{\mu}\left\{f_{2}(x) ; p, q ; \lambda ; \rho\right\}}\right) d x \leq \int_{0}^{b} \bar{p}(t) \Phi\left(\frac{f_{1}(t)}{f_{2}(t)}\right) d t
$$

holds true for all $f_{i} \in L^{1}[a, b]$.

Theorem 3.3. Let $\operatorname{Re}(p)>0, \operatorname{Re}(q)>0, \operatorname{Re}(\mu)>0, \operatorname{Re}(\lambda)>0$ and $\operatorname{Re}(\rho)>$ 0 . Let $D_{x}^{\mu}\{f(x) ; p, q, \lambda, \rho\}$ be the extension of extended Riemman-Liouvill fractional derivative of order $\mu$. Let $u$ be a weight function defined on $(0, b)$. Moreover, if $\Phi$ is a convex function on an interval $I \subseteq \mathbb{R}$ and $\varphi: I \rightarrow \mathbb{R}$ is any function, such that $\varphi(x) \in \partial \Phi(x)$ for all $x \in \operatorname{Int} I$ and $\bar{v}$ as in (3.2), then the inequality

$$
\begin{aligned}
& \int_{0}^{b} \bar{v}(t) \Phi(f(t)) d t-\int_{0}^{b} u(x) \Phi\left(\frac{\mathfrak{D}_{x}^{\mu}\{f(x) ; p, q ; \lambda ; \rho\}}{\bar{K}(x)}\right) d x \\
\geq & \frac{1}{\Gamma(-\mu)} \int_{0}^{b} \frac{u(x)}{\bar{K}(x)} \int_{a}^{x}(x-t)^{-\mu-1}{ }_{1} F_{1}\left[\lambda ; \rho ;-\frac{p x}{t}\right]{ }_{1} F_{1}\left[\lambda ; \rho ;-\frac{q x}{(x-t)}\right]
\end{aligned}
$$




$$
\begin{aligned}
& \times|| \Phi(f(t))-\Phi\left(\frac{\mathfrak{D}_{x}^{\mu}\{f(x) ; p, q ; \lambda ; \rho\}}{\bar{K}(x)}\right) \mid \\
& -\left|\varphi\left(\frac{\mathfrak{D}_{x}^{\mu}\{f(x) ; p, q ; \lambda ; \rho\}}{\bar{K}(x)}\right)\right| \cdot \mid f(t)-\left(\frac{\mathfrak{D}_{x}^{\mu}\{f(x) ; p, q ; \lambda ; \rho\}}{\bar{K}(x)}\right) \| d t d x
\end{aligned}
$$

holds for all measurable functions $f:(0, b) \rightarrow \mathbb{R}$, such that $f(t) \in I$ for all $t \in(a, b)$. If $\Phi$ is a monotone convex function on an interval $I \subseteq \mathbb{R}$, then the inequality

$$
\begin{aligned}
& \int_{0}^{b} \bar{v}(t) \Phi(f(t)) d t-\int_{0}^{b} u(x) \Phi\left(\frac{\mathfrak{D}_{x}^{\mu}\{f(x) ; p, q ; \lambda ; \rho\}}{\bar{K}(x)}\right) d x \\
\geq & \mid \frac{1}{\Gamma(-\mu)} \int_{0}^{b} \frac{u(x)}{\bar{K}(x)} \int_{a}^{x} \operatorname{sgn}\left(f(t)-\frac{\mathfrak{D}_{x}^{\mu}\{f(x) ; p, q ; \lambda ; \rho\}}{\bar{K}(x)}\right) \\
& \times(x-t)^{-\mu-1}{ }_{1} F_{1}\left[\lambda ; \rho ;-\frac{p x}{t}\right]{ }_{1} F_{1}\left[\lambda ; \rho ;-\frac{q x}{(x-t)}\right] \\
& \times \mid \Phi(f(t))-\Phi\left(\frac{\mathfrak{D}_{x}^{\mu}\{f(x) ; p, q ; \lambda ; \rho\}}{\bar{K}(x)}\right) \\
& \left.-\left|\varphi\left(\frac{\mathfrak{D}_{x}^{\mu}\{f(x) ; p, q ; \lambda ; \rho\}}{\bar{K}(x)}\right)\right| \cdot\left(f(t)-\frac{\mathfrak{D}_{x}^{\mu}\{f(x) ; p, q ; \lambda ; \rho\}}{\bar{K}(x)}\right)\right] d t d x \mid
\end{aligned}
$$

holds for all measurable functions $f:(0, b) \rightarrow \mathbb{R}$.

Proof. Similar to proof of Theorem 3.1, by applying Theorem 1.2.

Theorem 3.4. Let $\operatorname{Re}(p)>0, \operatorname{Re}(q)>0, \operatorname{Re}(\mu)>0, \operatorname{Re}(\lambda)>0$ and $\operatorname{Re}(\rho)>$ 0. Let $D_{x}^{\mu}\{f(x) ; p, q, \lambda, \rho\}$ be the extension of extended Riemman-Liouvill fractional derivative of order $\mu$, and I a compact interval of $\mathbb{R}, \tilde{h} \in C^{2}(I)$ and let $f:(0, b) \rightarrow \mathbb{R}$ be a measurable function such that $\operatorname{Im} f \subseteq I$. Then for the weight function u defined on $(0, b)$ there exists $\eta \in I$ such that

$$
\begin{aligned}
& \int_{0}^{b} \bar{v}(t) \tilde{h}(f(t)) d t-\int_{0}^{b} u(x) \tilde{h}\left(\frac{\mathfrak{D}_{x}^{\mu}\{f(x) ; p, q ; \lambda ; \rho\}}{\bar{K}(x)}\right) d x \\
= & \frac{\tilde{h}^{\prime \prime}(\eta)}{2}\left[\int_{0}^{b} \bar{v}(t) f^{2}(t) d t-\int_{0}^{b} u(x)\left(\frac{\mathfrak{D}_{x}^{\mu}\{f(x) ; p, q ; \lambda ; \rho\}}{\bar{K}(x)}\right)^{2} d x\right],
\end{aligned}
$$

where $\bar{v}$ is defined by (3.2).

Proof. Similar to proof of Theorem 3.1, by applying Theorem 1.3.

Theorem 3.5. Let $\operatorname{Re}(p)>0, \operatorname{Re}(q)>0, \operatorname{Re}(\mu)>0, \operatorname{Re}(\lambda)>0$ and $\operatorname{Re}(\rho)>0$. Let the extension of extended Riemman-Liouvill fractional derivative $D_{x}^{\mu}\{f(x) ; p, q, \lambda, \rho\}$ of order $\mu$ and $I$ a compact interval of $\mathbb{R}, k, \tilde{h} \in C^{2}(I)$ such that $\tilde{h}^{\prime \prime}(x) \neq 0$ for every 
$x \in I$. Moreover, $f:(0, b) \rightarrow \mathbb{R}$ a measurable function with $\operatorname{Im} f \subseteq I, u$ a weight function, $\bar{v}$ as in (3.2) and

$$
\int_{0}^{b} \bar{v}(t) \tilde{h}(f(t)) d t-\int_{0}^{b} u(x) \tilde{h}\left(\frac{\mathfrak{D}_{x}^{\mu}\{f(x) ; p, q ; \lambda ; \rho\}}{\bar{K}(x)}\right) d x \neq 0 .
$$

Then there exists $\eta \in I$ such that the following equality holds true

$$
\frac{k^{\prime \prime}(\eta)}{\tilde{h}^{\prime \prime}(\eta)}=\frac{\int_{0}^{b} \bar{v}(t) k(f(t)) d t-\int_{0}^{b} u(x) k\left(\frac{\mathfrak{D}_{x}^{\mu}\{f(x) ; p, q ; \lambda ; \rho\}}{\bar{K}(x)}\right) d x}{\int_{0}^{b} \bar{v}(t) \tilde{h}(f(t)) d t-\int_{0}^{b} u(x) \tilde{h}\left(\frac{\mathfrak{D}_{x}^{\mu}\{f(x) ; p, q ; \lambda ; \rho\}}{\bar{K}(x)}\right) d x} .
$$

Proof. Similar to proof of Theorem 3.1.

Theorem 3.6. Let $\operatorname{Re}(p)>0, \operatorname{Re}(q)>0, \operatorname{Re}(\mu)>0, \operatorname{Re}(\lambda)>0$ and $\operatorname{Re}(\rho)>0$. Let the fractional derivative operator $D_{x}^{\mu}\{f(x) ; p, q, \lambda, \rho\}$ of order $\mu$ and $f$ a positive function and let $u$ be a weight function defined on $(a, b), \bar{v}$ be as in (4.4). Then the function $\xi: \mathbb{R} \rightarrow[0, \infty)$ defined by

$$
\xi(s)=\int_{0}^{b} \bar{v}(t) \varphi_{s}(f(t)) d t-\int_{0}^{b} u(x) \varphi_{s}\left(\frac{\mathfrak{D}_{x}^{\mu}\{f(x) ; p, q ; \lambda ; \rho\}}{\bar{K}(x)}\right) d x
$$

is exponentially convex.

Proof. Similar to proof of Theorem 3.1, by applying Theorem 1.4.

4. Inequalities for Modified Extension of Riemman-Liouvill Fractional Derivative

The following definition is given in [18].

\section{Definition 4.1.}

$$
\mathfrak{D}_{z, p}^{\mu, \alpha}\{f(z)\}=\frac{1}{\Gamma(-\mu)} \int_{0}^{z} f(t)(z-t)^{-\mu-1} E_{\alpha}\left(-\frac{p z^{2}}{t(z-t)}\right) d t, \quad \operatorname{Re}(\mu)>0,
$$

where

$$
E_{\alpha}(z)=\sum_{n=0}^{\infty} \frac{z^{n}}{\Gamma(\alpha n+1)} .
$$

For the case $m-1<\operatorname{Re}(\mu)<m$, where $m=1,2, \ldots$, it follows

$$
\begin{aligned}
\mathfrak{D}_{z, p}^{\mu, \alpha}\{f(z)\} & =\frac{d^{m}}{d x^{m}} \mathfrak{D}_{z, p}^{\mu-m, \alpha}\{f(z)\} \\
& =\frac{d^{m}}{d x^{m}}\left\{\frac{1}{\Gamma(-\mu+m)} \int_{0}^{z} f(t)(z-t)^{-\mu+m-1} E_{\alpha}\left(-\frac{p z^{2}}{t(z-t)}\right) d t\right\},
\end{aligned}
$$

where $\operatorname{Re}(\mu)>0, \operatorname{Re}(p)>0, \operatorname{Re}(q)>0$. 
Remark 4.1. Obviously if $\alpha=1$, then (4.1) and (4.3) reduces to the extended fractional derivative (2.3) and (2.4), respectively. Similarly, if we set $\alpha=1$ and $p=0$, we get (2.1) and (2.2), respectively.

Very recently Shadab et al. [20] introduce new and modified extension of beta function as:

$$
B_{p}^{\alpha}\left(\sigma_{1}, \sigma_{2}\right)=\int_{0}^{1} t^{\sigma_{1}-1}(1-t)^{\sigma_{2}-1} E_{\alpha}\left(-\frac{p}{t(1-t)}\right),
$$

where $\operatorname{Re}\left(\sigma_{1}\right)>0, \operatorname{Re}\left(\sigma_{2}\right)>0$ and $E_{\alpha}(\cdot)$ is defined by (4.2).

Theorem 4.1. Let $\mathfrak{D}_{x, p}^{\mu, \alpha}\{f(z)\}$ denotes the new and modified extension of RiemannLiouville fractional derivative of order $\mu$ and let $u$ be a weight function defined on $(0, b)$, then $\hat{v}$ is defined by

$$
\hat{v}(t)=\int_{t}^{b} u(x) \frac{(x-t)^{-\mu-1} E_{\alpha}\left(-\frac{p z^{2}}{t(z-t)}\right)}{x^{-\mu} B_{p}^{\alpha}(1,-\mu)} d x<\infty .
$$

If $\Phi$ is a convex function on the interval $I$, then the inequality

$$
\int_{0}^{b} u(x) \Phi\left(\frac{\Gamma(-\mu) \mathfrak{D}_{x, p}^{\mu, \alpha}\{f(x)\}}{x^{-\mu} B_{p}^{\alpha}(1,-\mu)}\right) d x \leq \int_{0}^{b} \hat{v}(t) \Phi(f(t)) d t
$$

holds true.

Proof. Applying Theorem 1.1, with $\Omega_{1}=\Omega_{2}=(0, b), d \mu_{1}(x)=d x, d \mu_{2}(t)=d t$,

$$
\begin{aligned}
& \hat{k}(x, t)= \begin{cases}\frac{1}{\Gamma(-\mu)}(x-t)^{-\mu-1} E_{\alpha}\left(-\frac{p z^{2}}{t(z-t)}\right), & 0 \leq t \leq x, \\
0, & x<t \leq b,\end{cases} \\
& \hat{K}(x)=\frac{1}{\Gamma(-\mu)} \int_{0}^{x}(x-t)^{-\mu-1} E_{\alpha}\left(-\frac{p z^{2}}{t(z-t)}\right) d t=\frac{1}{\Gamma(-\mu)} x^{-\mu} B_{p}^{\alpha}(1,-\mu)
\end{aligned}
$$

and $\hat{v}$ as in (4.4), we get inequality (4.5).

Substitute $\hat{k}(x, t)$ by $\hat{k}(x, t) f_{2}(t)$ and $f$ by $\frac{f_{1}}{f_{2}}$, where $f_{i}: \Omega_{2} \rightarrow \mathbb{R}, i=1,2$, are measurable functions in Theorem 4.1 we obtain the following result.

Theorem 4.2. Let $\mathfrak{D}_{x, p}^{\mu, \alpha}\{f(z)\}$ denotes the new and modified extension of RiemannLiouville fractional derivative of order $\mu$ and let $u$ be a weight function defined on $(0, b)$ and for each fixed $t \in(0, b)$ define $\hat{p}$ on $(0, b)$ as

$$
\hat{p}(t):=\frac{f_{2}(t)}{\Gamma(-\mu)} \int_{t}^{b} u(x) \frac{(x-t)^{-\mu-1} E_{\alpha}\left(-\frac{p z^{2}}{t(z-t)}\right)}{\mathfrak{D}_{x, p}^{\mu, \alpha}\left\{f_{2}(x)\right\}} d x<\infty .
$$

If $\Phi: I \rightarrow \mathbb{R}$ is a convex function then the inequality

$$
\int_{0}^{b} u(x) \Phi\left(\frac{\mathfrak{D}_{x, p}^{\mu, \alpha}\left\{f_{1}(x)\right\}}{\mathfrak{D}_{x, p}^{\mu, \alpha}\left\{f_{2}(x)\right\}}\right) d x \leq \int_{a}^{b} \hat{p}(t) \Phi\left(\frac{f_{1}(t)}{f_{2}(t)}\right) d t
$$


holds true for all $f_{i} \in L^{1}[a, b]$.

Refinement of Theorem 4.1 is given in the upcoming theorem.

Theorem 4.3. Let $\mathfrak{D}_{x, p}^{\mu, \alpha}\{f(z)\}$ denotes the new and modified extension of RiemannLiouville fractional derivative of order $\mu$ and let $u$ be a weight function defined on $(a, b)$. Moreover, if $\Phi$ is a convex function on an interval $I \subseteq \mathbb{R}$ and $\varphi: I \rightarrow \mathbb{R}$ is any function, such that $\varphi(x) \in \partial \Phi(x)$ for all $x \in \operatorname{Int} I$ and $\hat{v}$ as in (4.4), then the inequality

$$
\begin{aligned}
& \int_{0}^{b} \hat{v}(t) \Phi(f(t)) d t-\int_{0}^{b} u(x) \Phi\left(\frac{\Gamma(-\mu) \mathfrak{D}_{x, p}^{\mu, \alpha}\{f(x)\}}{x^{-\mu} B_{p}^{\alpha}(1,-\mu)}\right) d x \\
\geq & \int_{0}^{b} \frac{u(x)}{x^{-\mu} B_{p}^{\alpha}(1,-\mu)} \int_{a}^{x}(x-t)^{-\mu-1} E_{\alpha}\left(-\frac{p z^{2}}{t(z-t)}\right) \\
& \times|| \Phi(f(t))-\Phi\left(\frac{\Gamma(-\mu) \mathfrak{D}_{x, p}^{\mu, \alpha}\{f(x)\}}{x^{-\mu} B_{p}^{\alpha}(1,-\mu)}\right) \mid \\
& -\left|\varphi\left(\frac{\Gamma(-\mu) \mathfrak{D}_{x, p}^{\mu, \alpha}\{f(x)\}}{x^{-\mu} B_{p}^{\alpha}(1,-\mu)}\right)\right| \cdot \mid f(t)-\left(\frac{\Gamma(-\mu) \mathfrak{D}_{x, p}^{\mu, \alpha}\{f(x)\}}{x^{-\mu} B_{p}^{\alpha}(1,-\mu)}\right) \| d t d x
\end{aligned}
$$

holds for all measurable functions $f:(0, b) \rightarrow \mathbb{R}$. If $\Phi$ is a monotone convex function on an interval $I \subseteq \mathbb{R}$, then the inequality

$$
\begin{aligned}
& \int_{0}^{b} \hat{v}(t) \Phi(f(t)) d t-\int_{0}^{b} u(x) \Phi\left(\frac{\Gamma(-\mu) \mathfrak{D}_{x, p}^{\mu, \alpha}\{f(x)\}}{x^{-\mu} B_{p}^{\alpha}(1,-\mu)}\right) d x \\
\geq & \mid \int_{0}^{b} \frac{u(x)}{x^{-\mu} B_{p}^{\alpha}(1,-\mu)} \int_{0}^{x} \operatorname{sgn}\left(f(t)-\frac{\Gamma(-\mu) \mathfrak{D}_{x, p}^{\mu, \alpha}\{f(x)\}}{x^{-\mu} B_{p}^{\alpha}(1,-\mu)}\right) \\
& \times(x-t)^{-\mu-1} E_{\alpha}\left(-\frac{p z^{2}}{t(z-t)}\right)\left[\Phi(f(t))-\Phi\left(\frac{\Gamma(-\mu) \mathfrak{D}_{x, p}^{\mu, \alpha}\{f(x)\}}{x^{-\mu} B_{p}^{\alpha}(1,-\mu)}\right)\right. \\
& \left.-\left|\varphi\left(\frac{\Gamma(-\mu) \mathfrak{D}_{x, p}^{\mu, \alpha}\{f(x)\}}{x^{-\mu} B_{p}^{\alpha}(1,-\mu)}\right)\right| \cdot\left(f(t)-\frac{\Gamma(-\mu) \mathfrak{D}_{x, p}^{\mu, \alpha}\{f(x)\}}{x^{-\mu} B_{p}^{\alpha}(1,-\mu)}\right)\right] d t d x \mid
\end{aligned}
$$

holds for all measurable functions $f:(0, b) \rightarrow \mathbb{R}$.

Proof. Same as proof of Theorem 4.1, by applying Theorem 1.2.

Next we give the mean value theorems.

Theorem 4.4. Let $\mathfrak{D}_{x, p}^{\mu, \alpha}\{f(z)\}$ denotes the new and modified extension of RiemannLiouville fractional derivative of order $\mu, I$ be a compact interval of $\mathbb{R}, \tilde{h} \in C^{2}(I)$ and let $f:(0, b) \rightarrow \mathbb{R}$ be a measurable function such that $\operatorname{Im} f \subseteq I$. Then for the weight 
function $u$ defined on $(0, b)$ there exists $\eta \in I$ such that

$$
\begin{aligned}
& \int_{0}^{b} \hat{v}(t) \tilde{h}(f(t)) d t-\int_{0}^{b} u(x) \tilde{h}\left(\frac{\Gamma(-\mu) \mathfrak{D}_{x, p}^{\mu, \alpha}\{f(x)\}}{x^{-\mu} B_{p}^{\alpha}(1,-\mu)}\right) d x \\
= & \frac{\tilde{h}^{\prime \prime}(\eta)}{2}\left[\int_{0}^{b} \hat{v}(t) f^{2}(t) d t-\int_{0}^{b} u(x)\left(\frac{\Gamma(-\mu) \mathfrak{D}_{x, p}^{\mu, \alpha}\{f(x)\}}{x^{-\mu} B_{p}^{\alpha}(1,-\mu)}\right)^{2} d x\right],
\end{aligned}
$$

where $\hat{v}$ is defined by (4.4).

Proof. Similar to proof of Theorem 4.1, by applying Theorem 1.3.

Theorem 4.5. Let $\mathfrak{D}_{x, p}^{\mu, \alpha}\{f(z)\}$ denotes the new and modified extension of RiemannLiouville fractional derivative of order $\mu, I$ be a compact interval of $\mathbb{R}, k, \tilde{h} \in C^{2}(I)$ such that $\tilde{h}^{\prime \prime}(x) \neq 0$ for every $x \in I$. Moreover $f:(a, b) \rightarrow \mathbb{R}$ a measurable function with $\operatorname{Im} f \subseteq I, u$ be a weight function, $\hat{v}$ as in (4.4) and

$$
\int_{0}^{b} \hat{v}(t) \tilde{h}(f(t)) d t-\int_{0}^{b} u(x) \tilde{h}\left(\frac{\Gamma(-\mu) \mathfrak{D}_{x, p}^{\mu, \alpha}\{f(x)\}}{x^{-\mu} B_{p}^{\alpha}(1,-\mu)}\right) d x \neq 0 .
$$

Then there exists $\eta \in I$ such that the following equality holds true

$$
\frac{k^{\prime \prime}(\eta)}{\tilde{h}^{\prime \prime}(\eta)}=\frac{\int_{0}^{b} \hat{v}(t) k(f(t)) d t-\int_{0}^{b} u(x) k\left(\frac{\Gamma(-\mu) \mathfrak{D}_{x, p}^{\mu, \alpha}\{f(x)\}}{x^{-\mu} B_{p}^{\alpha}(1,-\mu)}\right) d x}{\int_{0}^{b} \hat{v}(t) \tilde{h}(f(t)) d t-\int_{0}^{b} u(x) \tilde{h}\left(\frac{\Gamma(-\mu) \mathfrak{D}_{x, p}^{\mu, \alpha}\{f(x)\}}{x^{-\mu} B_{p}^{\alpha}(1,-\mu)}\right) d x} .
$$

Theorem 4.6. Let $\mathfrak{D}_{x, p}^{\mu, \alpha}\{f(z)\}$ denotes the new and modified extension of RiemannLiouville fractional derivative of order $\mu$ and let $u$ be a weight function defined on $(a, b), \hat{v}$ be as in (4.4). Then the function $\xi: \mathbb{R} \rightarrow[0, \infty)$ defined by

$$
\xi(s)=\int_{0}^{b} \hat{v}(t) \varphi_{s}(f(t)) d t-\int_{0}^{b} u(x) \varphi_{s}\left(\frac{\Gamma(-\mu) \mathfrak{D}_{x, p}^{\mu, \alpha}\{f(x)\}}{x^{-\mu} B_{p}^{\alpha}(1,-\mu)}\right) d x
$$

is exponentially convex.

Proof. Similar to proof of Theorem 4.1, by applying Theorem 1.4.

Now we shall discuss the exponentially convexity of the liner functional. Under the assumptions of the Theorem 2.1, Theorem 3.1 and Theorem 4.1 we define a linear functionals by taking the positive difference of the inequalities stated in (2.7), (3.3) and (4.5), respectively as:

$$
\begin{aligned}
& \xi_{1}(\Phi)=\int_{0}^{b} \tilde{v}(t) \Phi(f(t)) d t-\int_{0}^{b} \Phi\left(\frac{\Gamma(-\mu) x^{\mu} \mathfrak{D}_{x}^{\mu}\{f(x) ; p, q\}}{B_{p, q}(1,-\mu)}\right) u(x) d x, \\
& \xi_{2}(\Phi)=\int_{0}^{b} \bar{v}(t) \Phi(f(t)) d t-\int_{0}^{b} \Phi\left(\frac{\mathfrak{D}_{x}^{\mu}\{f(x) ; p, q ; \lambda ; \rho\}}{\bar{K}(x)}\right) u(x) d x
\end{aligned}
$$


and

$$
\xi_{3}(\Phi)=\int_{0}^{b} \hat{v}(t) \Phi(f(t)) d t-\int_{0}^{b} \Phi\left(\frac{\Gamma(-\mu) \mathfrak{D}_{x, p}^{\mu, \alpha}\{f(x)\}}{x^{-\mu} B_{p}^{\alpha}(1,-\mu)}\right) u(x) d x .
$$

We also define a linear functionals by taking the positive difference of the left-hand side and right-hand side of the inequalities (2.9), (3.4) and (4.6), respectively as:

$$
\begin{aligned}
& \xi_{4}(\Phi)=\int_{0}^{b} \tilde{p}(t) \Phi\left(\frac{f_{1}(t)}{f_{2}(t)}\right) d t-\int_{0}^{b} u(x) \Phi\left(\frac{\mathfrak{D}_{x}^{\mu}\left\{f_{1}(x) ; p, q\right\}}{\mathfrak{D}_{x}^{\mu}\left\{f_{2}(x) ; p, q\right\}}\right) d x \\
& \xi_{5}(\Phi)=\int_{0}^{b} \bar{p}(t) \Phi\left(\frac{f_{1}(x)}{f_{2}(x)}\right) d t-\int_{0}^{b} u(x) \Phi\left(\frac{\mathfrak{D}_{x}^{\mu}\left\{f_{1}(x) ; p, q, \lambda, \rho\right\}}{\mathfrak{D}_{x}^{\mu}\left\{f_{2}(x) ; p, q, \lambda, \rho\right\}}\right) d x
\end{aligned}
$$

and

$$
\xi_{6}(\Phi)=\int_{0}^{b} \hat{p}(t) \Phi\left(\frac{f_{1}(x)}{f_{2}(x)}\right) d t-\int_{0}^{b} u(x) \Phi\left(\frac{\mathfrak{D}_{x}^{\mu}\left\{f_{1}(x) ; p, q\right\}}{\mathfrak{D}_{x}^{\mu}\left\{f_{2}(x) ; p, q\right\}}\right) d x .
$$

Theorem 4.7. Let $\Gamma=\left\{\Phi_{p}: p \in J\right\}$ be a family of functions defined on $I$, such that the function $p \mapsto\left[z_{0}, z_{1}, z_{2} ; \Phi_{p}\right]$ is n-exponentially convex in the Jensen sense on $J$ for every three distinct points $z_{0}, z_{1}, z_{2} \in I$. Let $\xi_{i}, i=1,2, \ldots, 6$, be linear functionals defined by (4.7)-(4.10), respectively. Then the function $p \mapsto \xi_{i}\left(\Phi_{p}\right), i=1,2, \ldots, 6$, is n-exponentially convex in the Jensen sense on J. If the function $p \mapsto \xi_{i}\left(\Phi_{p}\right)$ is continuous on $J$, then it is n-exponentially convex on $J$.

Acknowledgements. The research of author Josip Pečarić was supported by the Ministry of Education and Science of the Russian Federation (Agreement number No. 02.a03.21.0008). Authors are thankful to the referee and editor for their valuable suggestions, which improved the final version of this paper.

\section{REFERENCES}

[1] E. Adeleke, A. Čižmešija, J. Oguntuase, L. E. Persson and D. Pokaz, On a new class of Hardy-type inequalities, J. Inequal. Appl. 259 (2012).

[2] D. Baleanu, P. Agarwal, R. K. Parmar, M. M. Alquarashi and S. Salahshour, Extension of the fractional derivative operator of the Riemann-Liouville, J. Nonlinear Sci. Appl. 10 (2017), 2914-2924.

[3] S. N. Bernstein, Sur les fonctions absolument monotones, Acta Math. 52 (1929), 1-66.

[4] A. Čižmešija, K. Krulić and J. Pečarić, Some new refined Hardy-type inequalities with kernels, J. Math. Inequal. 4(4) (2010), 481-503.

[5] N. Elezović, K. Krulić and J. Pečarić, Bounds for Hardy type differences, Acta Math. Sin. (Engl. Ser.) 27(4) (2011), 671-684.

[6] S. Iqbal, J. Pečarić and Y. Zhou, Generalization of an inequality for integral transforms with kernel and related results, J. Inequal. Appl. 2010 (2010), Article ID 948430.

[7] S. Iqbal, K. Krulić, J. Pečarić and Dora Pokaz, n-Exponential convexity of Hardy-type and Boas-type functionals, J. Math. Inequal. 7(4) (2011), 739-750. 
[8] S. Iqbal, J. Pečarić, M. Samraiz and N. Sultana, Applications of refined Hardy-type inequalities, Math. Inequal. Appl. 18(4) (2015), 1539-1560.

[9] S. Iqbal, J. Pečarić, M. Samraiz and Z. Tomovski, Hardy-type inequalities for generalized fractional integral operators, Tbilisi Math. J. 10(1) (2017), 1-16.

[10] A. A. Kilbas, H. M. Sarivastava and J. J. Trujillo, Theory and Application of Fractional Differential Equations, North-Holland Mathematics Studies, Elsevier Sciences B.V., Amsterdam, 2006.

[11] K. Krulić, J. Pečarić and L. E. Persson, Some new Hardy type inequalities with general kernels, Math. Inequal. Appl. 12 (2009), 473-485.

[12] K. Mehrez and Z. Tomovski, On a new $(p, q)$-Mathieu type power series and its applications, Appl. Anal. Discrete Math. (2019) (to appear).

[13] C. Niculescu and L. E. Persson, Convex Functions and Their Applications. A Contemporary Approach, CMC Books in Mathematics Springer, New York, 2006.

[14] M. A. Ozerslan and E. Ozergin, Some generating relations for extended hypergeometric functions via generalized fractional derivative operator, Math. Comput. Model. 52 (2010), 1825-1833.

[15] J. Pečarić and J. Perić, Improvements of the Giaccardi and the Petrović inequality and related Stolarsky type means, An. Univ. Craiova Ser. Mat. Inform. 39(1) (2012), 65-75.

[16] L. E. Persson, M. A. Ragusa. N. Samko and P. Wall, Commutators of Hardy operators in vanishing Morrey spaces, American Institute of Physics 1493 (2012), 859-866.

[17] G. Rahman, S. Mubeen, K. S. Nisar and J. Choi, Certain extended special functions and fractional integral operator via an extended beta function, Nonlinear Functional Analysis and Applications 4(1) (2019), 1-13.

[18] G. Rahman, K. S. Nisar and Z. Tomovski, A new extention of Riemann-Liouville fractional derivative operator, Commun. Korean Math. Soc. 34(2) (2019), 507-522.

[19] S. G. Samko, A. A. Kilbas and O. I. Marichev, Fractional Integrals and Derivatives, Theory and Applications, Gordon and Breach Science Publishers, Yverdon, 1993.

[20] M. Shadab, S. Jabee and J. Choi, An extension of beta function and its application, Far East J. Math. Sci. 103(1) (2018), 235-251.

${ }^{1}$ Department of Mathematics, University of SARgodha (Sub-Campus BhakKar), BHAKKAR, PAKISTAN

Email address: sajid_uos2000@yahoo.com

Email address: sajid.iqbal@uos.edu.pk

${ }^{2}$ Department of Mathematics,

COMSATS Institute of Information TeChnology,

Attock, Pakistan

Email address: faridphdsms@hotmail.com

${ }^{3}$ RUDN UNIVERSITY,

Moscow, Russia

Email address: pecaric@element.hr

${ }^{4}$ Department of Mathematics,

Faculty of Technical Science, University Ismail Qemali,

Vlora, Albania

Email address: artionkashuri@gmail.com 